\title{
Added mass of an oscillating hemisphere at very-low and very-high frequencies
}

\author{
Mario A. Storti *and Jorge D’Elía ${ }^{\dagger}$ \\ Centro Internacional de Métodos Computacionales en Ingeniería (CIMEC) \\ INTEC, UNL-CONICET. Güemes 3450, 3000-Santa Fe, Argentina \\ http://www.cimec.org.ar, e-mail: cimec@ceride.gov.ar \\ ph: +54 3424556673 , fx: +543424550944
}

\begin{abstract}
A floating hemisphere under forced harmonic oscillation at very-low and very-high frequencies is considered. The problem is reduced to an elliptic one, that is, the Laplace operator in the exterior domain with Dirichlet and Neumann boundary conditions. Asymptotic values of the added mass are found with an analytic prolongation for the surge mode, and with a semi-numerical computation with spherical harmonics for the heave mode. The general procedure is based on the use of spherical harmonics and its derivation is based on a physical insight rather than a mathematical one. This case can be used to test the accuracy achieved by numerical codes based on other formulations as finite or boundary elements.
\end{abstract}

\section{Introduction}

This work is concerned with a computation of the added mass of a floating hemisphere due to a forced oscillatory motion of the body in the free surface of an inviscid incompressible fluid. Two canonical cases are considered, namely those of heave and surge motions, where these nautical terms are used to describe a vertical or horizontal oscillation of the body.

The present problem has several applications. The oscillation of a floating body implies wave radiation so that it is of interest to wave energy conversion (e.g. see Falnes ${ }^{19}$ ). In seakeeping

\footnotetext{
${ }^{*}$ Conicet Senior Researcher mstorti@intec.unl.edu.ar

${ }^{\dagger}$ Conicet Graduate Researcher jdelia@intec.unl.edu.ar
} 
hydrodynamics (e.g. see Ohkusu, ${ }^{24}$ Huang/Sclavounos ${ }^{\sqrt{36}}$ ), the hydrodynamic characteristics of a vessel due to action of surface waves when the wave motion is small enough compared to the body length can be computed with a linearized theory. Then, the response of a body to incident waves can be determined from the added-mass and damping coefficients associated to the forced motions of the body in the absence of waves, such as the heave motions of a semisubmersible platform due to the influence of ocean waves (see Hulme ${ }^{2}$ ). Besides, added mass effects can be recognized in ship vibrations. Although Lamb ${ }^{13}$ investigated the accelerated motion of a submerged cylinder, its relevance in ship vibrations was only properly recognized from the experimental work of Nicholls ${ }^{12}$ and the mathematical one (using conformal mapping) of Lewis $\frac{10}{}$.

From a physical point of view, the added mass effect comes from the inertia of the fluid. For instance, when a floating ship-like body performs a heave motion and the fluid is assumed as incompressible, there will be fluid motion between the hull and the free surface on the downbeat and back again on the upbeat, see Fig. 1. The added mass effects in this case come from the pressures transmitted to the hull arising from the inertia of the fluid. A similar analysis can be made for the surge motion, e.g. see Llloyd $\frac{\sqrt{6}}{\text { or Jennings }}{ }^{3}$.

The formulation of problems involving floating hemispheres is analogous to that for the corresponding two-dimensional ones involving circular cylinders and perhaps, as Hulme ${ }^{11}$ says, this is the reason why they have received rather comparatively little attention in the literature. The modern history of this subject began with Ursell ${ }^{8}$, who formulated and solved the boundary value problem for a semi-immersed heaving circular cylinder, where the velocity potential is represented as the sum of an infinite set of multipoles, each satisfying linearized free-surface boundary condition and each being multiplied by a coefficient determined by requiring the series to satisfy the kinematic boundary condition at a finite number of points on the cylinder. Grim ${ }^{28}$ used a variation of the Ursell method to solve the two-parameter Lewis-form cylinders by conformal mapping onto a circle. Tasai ${ }^{7}$ and Porter ${ }^{35}$ solved the added mass and damping using the Ursell approach for oscillating contours mappable onto a circle by the more general Theodorsen transformation, whereas Ogilvie ${ }^{31}$ computed the hydrodynamics forces on a com- 
pletely submerged heaving circular cylinder. Frank ${ }^{34}$ used an integral formulation where the velocity potential is represented by a distribution of sources over the submerged cross section; the density of the sources is an unknown function of the position along the contour to be determined from integral equations found by applying the kinematic boundary condition on the submerged part of the cylinder.

Havelock $^{33}$ gives an account of the heaving-hemisphere case, where the solution is found with a method similar to that used by Ursell for the circular cylinder, that is, the velocity potential is expressed in spherical polar coordinates as the sum of a wave source at the sphere center together with an infinite series of wave-free potentials. Then, the velocity potential satisfies all the boundary conditions except for the body surface, and the latter is used to generate an infinite linear system of equations for the infinite number of unknowns appearing in the expansion of the velocity potential. Hulme ${ }^{1}$ used an essentially equivalent approach to Havelock's but with several modifications and more rigorous justification, where the solution is found by means of an expansion for the velocity potential in terms of an infinity series of spherical harmonics from which the relevant forces may be computed.

As it is usual in seakeeping flow problems, the forces exerted on oscillating bodies are given as the added mass and damping coefficients, which measure the components of the wave force in phase with the acceleration and velocity of the body respectively. In this work only the former case is considered. The proposed method is almost exact in the sense that the solution can be done with very high precision, and the results can be used to determine the accuracy achieved by other methods, e.g. finite or boundary elements, which can be used to treat more general body geometries, e.g. see Nigrc ${ }^{25}$ et al.. Storti ${ }^{26 \mid 27}$ et al., D'Elía ${ }^{17}[18 \mid 16]$ et al..

In this work, the heave and surge modes at very low and very high frequencies of the unit hemisphere are reformulated as boundary value problems extended to all the space, they are solved by orthogonal expansion by means of spherical harmonics. In Hulme's work, the derivation of the asymptotic form for the surge-mass coefficient at very high frequencies $(\omega \rightarrow \infty)$ is, as Hulme says, suggestive rather than conclusive, so an alternative derivation is given here for the same coefficient where the present estimation is closely related to Hulme's estimate. 


\section{The oscillating unity hemisphere}

An oscillating unity hemisphere in a forced motion is considered. The unit hemisphere is the open surface $r=1,0 \leq \theta \leq \pi / 2,0 \leq \varphi \leq 2 \pi$, where $r$ is the radius, $\theta$ is the azimuthal angle and $\varphi$ is the circumferential one. Its edge is on the free surface of an irrotational and incompressible fluid without a mean flow, the fluid depth is assumed as infinity, the $z$-axis positive downward and the hydrostatic equilibrium plane is $z=0$, see Figs. 2 and 3 . Due to the symmetry, the spherical coordinates $(r, \theta, \varphi)$ are employed. In this work a whole linearized analysis is performed and for this reason the linearized surface boundary condition at $z=0$ is only used. As it is well known (e.g. see Newman ${ }^{21}$, Ogilvie ${ }^{32}$, Ohkusu ${ }^{24}$, Stoker ${ }^{20}$ ), the standard free surface boundary condition of linearized water-wave theory for sinusoidal time dependence is given by

$$
\phi_{, n}=\frac{\omega^{2}}{g} \phi \quad \text { at } z=0
$$

where $\phi$ is the velocity potential, $\phi_{, n} \equiv \partial \phi / \partial n$ is the normal velocity, $n$ is the unit normal, $z=0$ is the hydrostatic free surface, $\omega$ is the circular frequency of the sinusoidal oscillation, and $g$ is the gravity acceleration.

\subsection{Limits at very-low and very-high frequencies}

In both limit processes $\omega \rightarrow 0$ and $\omega \rightarrow \infty$ for the linearized free-surface boundary condition given by Eq. 11, it will be assumed that the velocity potential $\phi$, the normal velocity $\phi_{, n}$ and the gravity acceleration $g$ remain finite and bounded. Then, on the one hand, at very-low frequencies $(\omega \rightarrow 0)$ the free-surface boundary Eq. 1 shrinks to the homogeneous Neumann one $\phi_{, n}=0$ at $z=0$, that is,

$$
\omega \rightarrow 0:\left\{\begin{array}{lr}
\Delta \phi=0 & \text { in } \Omega ; \\
\phi_{, n}=0 & \text { at } z=0 ; \\
\phi_{, n}=h & \text { at } \Gamma_{b} ; \\
|\phi| \rightarrow 0 & \text { for }|x| \rightarrow \infty
\end{array}\right.
$$


On the other hand, at very-high frequencies $(\omega \rightarrow \infty)$ the free-surface boundary Eq. 1 shrinks to the homogeneous Dirichlet one $\phi=0$ at $z=0$, then

$$
\omega \rightarrow \infty:\left\{\begin{array}{rrr}
\Delta \phi=0 & \text { in } \Omega ; \\
\phi=0 & \text { at } z=0 ; \\
\phi_{, n}=h & \text { at } \Gamma_{b} ; \\
|\phi| \rightarrow 0 & \text { for }|\boldsymbol{x}| \rightarrow \infty
\end{array}\right.
$$

In Eq. 2 243, $\Delta$ is the Laplace operator, $h=h(\theta, \varphi)$ is the load given by the normal displacement of the mode under consideration, and the last condition is the radiation boundary condition at infinity. For simplicity, it is assumed that the load $h=h(\theta, \phi)$ is real, that is, the body motion is in phase with the fluid velocity. It should be noted that the original linearized flow problem is defined only in the lower region $z \leq 0$ but the analysis performed for the two limit processes of the linearized surface boundary condition at $z=0$, that is, for $\omega \rightarrow 0$ [Very Low Frequencies $(\mathrm{VLF})]$ and for $\omega \rightarrow \infty$ [Very High Frequencies (VHF)], suggests that the boundary conditions $\phi_{, n}=0$ at VLF and $\phi=0$ at VHF respectively could be taken into account at the plane $z=0$.

\subsection{The heave and surge modes}

The heave-mode excitation of the body (vertical oscillation) produces a symmetrical displacement around the vertical axis $z$ and it can be written as $h=\cos \theta$, with $0 \leq \theta \leq \pi / 2$, see Fig. 4. Analogously, the surge-mode excitation (horizontal oscillation) produces an antisymmetrical displacement with respect to the plane $x=0$ and it can be written as $h=\sin \varphi$, with $0 \leq \varphi<2 \pi$, see Fig. 5. Once the velocity potential $\phi$ is solved for each mode (i.e. the surge $\phi^{1}$ and the heave $\phi^{3}$ ones), the added mass $A_{i j}$ in the $i$ degree of freedom due to a harmonic unity excitation on the $j$-direction is computed as the surface integral

$$
A_{i j}=-\rho \int_{\Gamma_{b}} d \Gamma \phi^{i} \phi_{, n}^{j}
$$

over the body surface $\Gamma_{b}$, where $i=j=1$ for the surge mode and $i=j=3$ for the heave one, e.g. see Ohkusu, ${ }^{24}$ Newman. ${ }^{21}$ Then, the added mass coefficient is obtained as $A_{i j}^{\prime}=A_{i j} /(\rho V)$, where $V=(2 / 3) \pi R^{3}$ is the body volume of the hemisphere and $\rho$ is the fluid density. 


\section{The extended flow problems}

By symmetry, Eqn. 2/3 can be reproduced extending the flow problem to upper region $z<0$ by means of a reflection with respect to the plane $z=0$ and extending the load $h=h(\theta, \phi)$ in an appropriate way. For instance, the homogeneous Neumann boundary condition is obtained when the load $h$ is extended in a symmetrical way, i.e. $h(x, y, z)=h(x, y,-z)$, while the homogeneous Dirichlet one is obtained when the load $h$ is extended in an anti-symmetrical way, i.e. $h(x, y, z)=-h(x, y,-z)$. It should be noted that the plane $z=0$ is not really necessary in the subsequent analysis since the surface load extension automatically satisfied the suggested linearized boundary conditions $\phi_{, n}=0$ at VLF and $\phi=0$ at VHF, respectively.

\subsection{The extended heave-modes at VLF and VHF}

The extended heave loads at Very Low Frequencies (VLF) and Very High Frequencies (VHF) are obtained from the sphere ones as

$$
\left\{\begin{array}{l}
h=|\cos \theta| \quad \text { for low frequencies }(\omega \rightarrow 0) \\
h=\cos \theta \quad \text { for high frequencies }(\omega \rightarrow \infty)
\end{array}\right.
$$

where now $0 \leq \theta \leq \pi$ due to the extension to upper region $z<0$. Then, the boundary value problem of the heave-mode at very-low frequencies $(\omega \rightarrow 0)$ is written as

$$
\text { extended heave-mode at VLF }(\omega \rightarrow 0):\left\{\begin{array}{lr}
\Delta \phi=0 & \text { in } \Omega^{\prime} ; \\
\phi_{, n}=|\cos \theta| & \text { at } \Gamma_{b}^{\prime} ; \\
\phi_{, n}=0 & \text { at } z=0 ; \\
|\phi| \rightarrow 0 & \text { for }|\boldsymbol{x}| \rightarrow \infty ;
\end{array}\right.
$$

see Fig. 6, where $\Omega^{\prime}=\Omega_{e} \cup \Omega_{e}^{\prime}$ is the extended flow domain, $\Omega_{e}$ and $\Omega_{e}^{\prime}$ are the flow domain exterior to the hemisphere and its extension through the reflection plane $z=0$, respectively, and $\Gamma_{b}^{\prime}$ is extended hemisphere surface through the same plane. In general, due to the module on source term $|\cos \theta|$, this case does not have a closed solution. Therefore it must be found with other resources like spherical harmonics, as considered in this work. 
Analogously, the boundary value problem of the heave-mode at very-high frequencies $(\omega \rightarrow \infty)$ is written as

$$
\text { extended heave-mode at VHF }(\omega \rightarrow \infty):\left\{\begin{array}{cr}
\Delta \phi=0 & \text { in } \Omega^{\prime} ; \\
\phi_{, n}=\cos \theta & \text { at } \Gamma_{b}^{\prime} ; \\
\phi=0 & \text { at } z=0 ; \\
|\phi| \rightarrow 0 & \text { for }|x| \rightarrow \infty ;
\end{array}\right.
$$

see Fig. 7 where, since the free surface boundary condition for VHF is $\phi=0$, its right hand side term has been extended in an anti-symmetric way and, then, it is equivalent to a sphere in infinite medium.

\subsection{The extended surge-mode}

On the other hand, the extended surge loads at the VLF and VHF, e.g. see Fig. 8 and 9, are

$$
\begin{cases}h=\sin \varphi \sin \theta & \text { for low frequencies }(\omega \rightarrow 0) \\ h=\sin \varphi \sin \theta \operatorname{sign}\{\cos \theta\} & \text { for high frequencies }(\omega \rightarrow \infty) .\end{cases}
$$

Then, the boundary value problem of the surge-mode at very-low frequencies $(\omega \rightarrow 0)$ is written as

$$
\text { extended surge-mode at VLF }(\omega \rightarrow 0):\left\{\begin{array}{lr}
\Delta \phi=0 & \text { in } \Omega^{\prime} ; \\
\phi_{, n}=\sin \varphi \sin \theta & \text { at } \Gamma_{e}^{\prime} ; \\
\phi_{, n}=0 & \text { at } z=0 ; \\
|\phi| \rightarrow 0 & \text { for }|\boldsymbol{x}| \rightarrow \infty ;
\end{array}\right.
$$

and the boundary value problem at very-high frequencies $(\omega \rightarrow \infty)$ is written as

$$
\text { extended surge-mode at } \operatorname{VHF}(\omega \rightarrow \infty): \begin{cases}\Delta \phi=0 & \text { in } \Omega^{\prime} ; \\ \phi_{, n}=\sin \varphi \sin \theta \operatorname{sign}\{\cos \theta\} & \text { at } \Gamma_{e}^{\prime} ; \\ \phi=0 & \text { at } z=0 ; \\ |\phi| \rightarrow 0 & \text { for }|\boldsymbol{x}| \rightarrow \infty .\end{cases}
$$




\section{Solution of the extended flow problems}

The extended flow problems can be solved in an analytical way or by series. The solutions for the extended flow problems corresponding to the heave-mode at VHF $(\omega \rightarrow \infty)$ and surge-mode at VLF $(\omega \rightarrow 0)$, are the same of a sphere in an infinity medium and uniform velocity, so the additional mass is half the displaced mass, that is,

$$
\left\{\begin{array}{rl}
A_{33}(\omega \rightarrow \infty) & =\frac{\pi}{3} \rho R^{3} \\
A_{11}(\omega \rightarrow 0) & =\frac{\pi}{3} \rho R^{3}
\end{array} .\right.
$$

In the other two cases, the heave-mode at VLF $(\omega \rightarrow 0)$ and the surge-mode at VHF $(\omega \rightarrow \infty)$, are not that easy to obtain, so the solutions are found expanding the sources by means of spherical harmonics.

\section{$5 \quad$ Spherical harmonics}

The exterior potential problem

$$
\begin{cases}\Delta \phi=0 & \text { for } r>1 ; \\ \phi=f(\theta, \varphi) & \text { at } r=1 ;\end{cases}
$$

where $\phi=\phi(\theta, \varphi)$ is solved expanding the function $f(\theta, \varphi)$ in terms of the harmonics

$$
\begin{aligned}
f(\theta, \varphi) & =\sum_{n=0}^{\infty} a_{n 0} P_{n}(\cos \theta) \\
& +\sum_{n=0}^{\infty} \sum_{m=1}^{n}\left[a_{n m} \cos (m \varphi)+b_{n m} \sin (m \varphi)\right] P_{n}^{m}(\cos \theta)
\end{aligned}
$$

where $P_{n}(u)$ are the Legendre polynomials, with $u=\cos \theta, P_{n}^{m}(u)=\left(1-u^{2}\right)^{m / 2} d^{m} P_{n} / d u^{m}$ are the associated ones, while the coefficients are given by

$$
\begin{gathered}
a_{n 0}=\frac{2 n+1}{4 \pi} \int_{r=1} d \Gamma f(\theta, \varphi) P_{n}(\cos \theta) \\
a_{n m}=\frac{2 n+1}{2 \pi} \frac{(n-m) !}{(n+m) !} \int_{r=1} d \Gamma f(\theta, \varphi) P_{n}^{m}(\cos \theta) \cos m \varphi \\
b_{n m}=\frac{2 n+1}{2 \pi} \frac{(n-m) !}{(n+m) !} \int_{r=1} d \Gamma f(\theta, \varphi) P_{n}^{m}(\cos \theta) \sin m \varphi
\end{gathered}
$$


where $d \Gamma=\sin \theta d \varphi d \theta$ is the differential of the solid angle in spherical coordinates. Once this expansion is computed, the exterior potential can be written as

$$
\phi(r, \theta, \varphi)=\sum_{n=0}^{\infty} Y_{n}(\theta, \varphi) r^{-(n+1)}
$$

where

$$
Y_{n}(\theta, \varphi)=a_{n 0} P_{n}(\cos \theta)+\sum_{m=1}^{n}\left[a_{n m} \cos (m \varphi)+b_{n m} \sin (m \varphi)\right] P_{n}^{m}(\cos \theta) .
$$

Then, the Neumann problem can be solved taking derivatives with respect to $r$ and evaluating at $r=1$, that is, $\left.h \equiv \phi_{, r}\right|_{r=1}$, obtaining the expression

$$
h(\theta, \varphi)=-\sum_{n=0}^{\infty}(n+1) Y_{n}(\theta, \varphi)
$$

from which analogous relations are obtained

$$
\begin{gathered}
a_{n 0}=\frac{2 n+1}{4 \pi(n+1)} \int_{r=1} d \Gamma h(\theta, \varphi) P_{n}(\cos \theta) ; \\
a_{n m}=\frac{2 n+1}{2 \pi(n+1)} \frac{(n-m) !}{(n+m) !} \int_{r=1} d \Gamma h(\theta, \varphi) P_{n}^{m}(\cos \theta) \cos (m \varphi) ; \\
b_{n m}=\frac{2 n+1}{2 \pi(n+1)} \frac{(n-m) !}{(n+m) !} \int_{r=1} d \Gamma h(\theta, \varphi) P_{n}^{m}(\cos \theta) \sin (m \varphi) .
\end{gathered}
$$

Once the coefficients of the expansion are obtained, the added mass can be found from

$$
A_{j j}=-\rho \int_{r=1} d \Gamma \phi \phi_{, r}=-\rho \int_{0}^{2 \pi} G(\varphi) d \varphi \int_{0}^{\pi} H(\theta) d \theta
$$

where $G(\varphi)=\left\{1, \sin ^{2} \varphi\right\}$ for the heave and surge motions, respectively, and

$$
H(\theta)=\sum_{n=0}^{\infty}\left\{2 a_{n 0}^{2}\left[P_{n}(\cos \theta)\right]^{2}+\sum_{m=1}^{n}\left(a_{n m}^{2}+b_{n m}^{2}\right) \times\left[P_{n}^{m}(\cos \theta)\right]^{2}\right\} ;
$$

where the orthogonality property of the spherical harmonics was taken into account. Finally, using properties of the Legendre polynomials

$$
A_{j j}=\rho \alpha \sum_{n=1}^{\infty} \frac{n+1}{2 n+1}\left[2 a_{n 0}^{2}+\sum_{m=1}^{n}\left(a_{n m}^{2}+b_{n m}^{2}\right) \frac{(n+m) !}{(n-m) !}\right]
$$

where $\alpha=\{2 \pi, \pi\}$, for the heave and surge motions, respectively. 


\section{Hemisphere in heave-mode at very-low frequencies}

The load in the heave-mode at $\operatorname{VLF}$ is $h(\theta, \varphi)=|\cos \theta|$, so

$$
a_{n 0}=\frac{2 n+1}{4 \pi(n+1)} \int_{-1}^{1}|\mu| P_{n}(\mu) d \mu \int_{0}^{2 \pi} d \varphi
$$

where $\mu=\cos \theta$. As the $P_{n}(\mu)$ are even (odd) for $n$ even (odd), only remains its even terms and then

$$
a_{n 0}=\frac{2 n+1}{n+1} \int_{0}^{1} \mu P_{n}(\mu) d \mu
$$

for $n$ even. For computing this integral, the $P_{n}$ terms are generated in a recursive way with the initial conditions $P_{0}=1, P_{1}=\mu$, and the next terms $P_{2}, \ldots, P_{n}$ are obtained by solving

$$
(n+1) P_{n+1}-(2 n+1) \mu P_{n}(\mu)+n P_{n-1}(\mu)=0 .
$$

The coefficients of the polynomials $\mu P_{n}(\mu)$ are obtained from the $P_{n}(\mu)$ ones, and the integral is made in a semi-analytical way. The final result for the added mass in the heave-mode at VLF for the sphere is

$$
A_{33}(\omega \rightarrow 0)=1.740335785143 \rho R^{3}
$$

corresponding to $A_{33}^{\prime}(\omega \rightarrow 0)=0.830949128536$, that is, the non-dimensional coefficient with respect to the hemisphere mass $2 / 3 \pi \rho R^{3}$.

\section{Hemisphere in surge-mode at very-high frequencies}

In this case, due to the load $h=\sin \varphi \sin \theta \operatorname{sign}\{\cos \theta\}$, the only no-null coefficients are the $b_{n 1}$ terms. For obtaining them an integral from $\mu=-1$ to $\mu=1$ must be made, with a function which includes the $P_{n}^{1}(\mu)$ terms. These terms have a factor $\sqrt{1-\mu^{2}}$, so it is more convenient to perform a semi-numerical integration (e.g. see Appendix). The final result is

$$
A_{11}(\omega \rightarrow \infty)=0.570136261149 \rho R^{3}
$$

corresponding to $A_{11}^{\prime}(\omega \rightarrow \infty)=0.272220012$ 593, that is, the non-dimensional coefficient with respect to the hemisphere mass $(2 / 3) \pi \rho R^{3}$. 


\section{Discussion}

As it can be seen, the solutions to these problems have somewhat different properties according to whether the body oscillates in heave (vertically) or in surge (horizontally). On the one hand, at Very Low Frequencies (VLF), if the body oscillates in heave its image moves oppositely (see Fig. 6) so that the two act together somewhat as a pulsating source, while if the body oscillates in surge its image moves in the same direction (see Fig. 8) and the total effect on the pressure load is the same as for a horizontal dipole in an infinite fluid. On the other hand, at Very High Frequencies (VHF), if the body oscillates in heave its image moves in the same direction (see Fig. 7) so that the two act together somewhat as a vertical dipole, while if the body oscillates in surge its image moves oppositely (see Fig. 9) and the total effect on the pressure load is the same as a pair of horizontal dipoles oriented in opposite directions. Furthermore, the intensity of the velocity potentials $\phi^{j}$, with $j=1$ (surge) and $j=3$ (heave), are proportional to the source terms of the corresponding governing differential equations which, in turn, are fixed by these pressures loads. As the added mass is proportional to the integral of the fluid velocity at the surface of the extended body, then the resulting added mass can be expected to be greater for heave motion at Very Low Frequencies (VLF) (since its pressure load is always positive on the extended surface) than for the surge one at Very High Frequencies (VHF) (since its pressure load has both positive and negative values). In any case, the added mass at any frequency in heave motion is always greater than in the surge one (since the displaced fluid is greater in heave than in surge), as it is well known from the corresponding plots as functions of the frequency obtained, for instance, by numerical computations, e.g. see Papanikolau ${ }^{4}$ or D’Elía ${ }^{15}$. A comprehensive analysis of relations between added masses and sources and doublets are given, for example, by Ogilvie $^{32}$ and Landweber ${ }^{22}$.

The present estimates for the added mass coefficients with respect to the hemisphere mass $2 / 3 \pi \rho R^{3}$, for the surge mode $(i=1$, longitudinal oscillation) and for the heave one $(i=3$, vertical oscillation), at $\operatorname{VLF}(\omega \rightarrow 0)$ and $\operatorname{VHF}(\omega \rightarrow \infty)$ limits, are summarized in Table 1 and compared to some literature values found (i) for the surge/sway mode, e.g. see Sierevoge 23 , Prins ${ }^{14}$ (where only the intervals $[0.25,1.50]$ and $[0.6,1.5]$ are respectively considered and so the 
extrapolations are rather doubtful); (ii) for the heave one, e.g. see Korsmeyer ${ }^{111}$ and Liapis ${ }^{30}$; and (iii) Hulme ${ }^{1}$. The Sierevogel, Prins and Liapis results are obtained with a panel method and Kelvin source; Korsmeyer used a panel method with Fourier transform and complex impedance extended to very-low frequencies, while Hulme's numerical results are obtained by spherical harmonics but with a rather different derivation and implementation.

In Hulme's work, the solution for each mode is expressed in terms of infinite series of spherical harmonics and then it is found solving truncated infinite linear system of equations. Hulme notes that its procedure is successful for slow oscillations of the body, i.e. small $K a$, where $a$ is the hemi-sphere radius (here Hulme's notation is employed) but, for high frequencies $K a$ the system is ill-conditioned so, for such cases, the problems are re-formulated as integral equations whose kernels become small as $K a \rightarrow \infty$. This same procedure is also used by Ursell ${ }^{9}$ and Davis ${ }^{5}$ but, as a rigorous treatment of the surge case involves a significant amount of mathematical labour, Hulme gives a plausible procedure from which the surge-added mass coefficient is approximated as

$$
A^{(1)} \sim C_{1}-\frac{C_{2}}{K a} \quad \text { as } K a \rightarrow \infty
$$

with

$$
C_{1}=-\frac{3}{2} \sum_{n=1}^{\infty} \alpha_{n} I\{2 n, 1 ; 1\} \quad ; \quad C_{2}=+\frac{3}{2} \sum_{n=1}^{\infty} \beta_{n} I\{2 n, 1 ; 1\} ;
$$

(Eq. 5.14 to 5.16, p.460, op. cit.) where the terms in the series for $C_{1}$ and $C_{2}$ decay as $1 / n^{3}$ and $\log (n) / n^{2}$, respectively, so the $C_{2}$ coefficient is neglected by Hulme and, then, the surge-mass coefficient at VHF is assumed equal to $A^{(1)} \approx C_{1} \approx 0.273239 \ldots$ which is closely related to the present estimate.

As conclusion, an alternative derivation of the added mass coefficients for the heave and surge motions of the unit floating hemisphere at very-low and very-high frequencies was shown. As a particular case, the numerical value obtained by the present procedure for the surge-mass coefficient at very high frequencies $(\omega \rightarrow \infty)$ is closely related to Hulme's suggestive one. Other modeling efforts would be focused on its extension to other analytic body shapes which can be extended to the upper region as spheroids or ellipsoids. These simpler geometries can be used 
as test cases for validation of related numerical codes, as those used in ship-hydrodynamics or fluid-solid interaction.

\section{Acknowledgment}

This work was partially performed with the Free Software Foundation/GNU-Project resources, as GNU/Linux OS and GNU/Octave, and supported through grants CONICET-PIP-198/98 (Germen-CFD), SECyT-FONCyT-PICT-51 (Germen) and CAI+D-UNL-94-004-024. The authors thanks the referees for to notice an error in the original manuscript, their constructive suggestions and careful reading.

\section{Appendix}

The load in the surge-mode at VHF is $h=\sin \varphi \sin \theta \operatorname{sign}\{\cos \theta\}$. The only no-null coefficients are the $b_{n 1}$ terms, that is,

$$
b_{n 1}=\frac{2 n+1}{2 \pi} \frac{1}{n(n+1)^{2}} \int_{0}^{2 \pi} \sin ^{2} \varphi d \varphi \int_{0}^{\pi} \sin \theta \operatorname{sign}\{\cos \theta\} P_{n}^{1}(\cos \theta) \sin \theta d \theta ;
$$

introducing $\mu=\cos \theta$ and integrating in $\varphi$,

$$
b_{n 1}=\frac{2 n+1}{2} \frac{1}{n(n+1)^{2}} \int_{-1}^{1} \sqrt{1-\mu^{2}} \operatorname{sign}\{\mu\} P_{n}^{1}(\mu) d \mu
$$

only the even terms are no-nulls, so

$$
b_{n 1}=\frac{2 n+1}{n(n+1)^{2}} \int_{0}^{1}\left(1-\mu^{2}\right) \frac{d P_{n}}{d \mu} d \mu
$$

integrating by parts,

$$
b_{n 1}=\frac{2 n+1}{n(n+1)^{2}}\left[\left|\left(1-\mu^{2}\right) P_{n}(\mu)\right|_{0}^{1}+2 \int_{0}^{1} \mu P_{n}(\mu) d \mu\right] ;
$$

or

$$
b_{n 1}=\frac{2 n+1}{n(n+1)^{2}}\left[-P_{n}(0)+2 \int_{0}^{1} \mu P_{n}(\mu) d \mu\right]
$$

which is computed with a semi-numerical procedure, for instance, with Octave 29 .

\section{References}

[1] Hulme A. The wave forces acting on a floating hemisphere undergoing forced periodic oscillations. J. Fluid Mechanics, 121:443-463, 1982. 
[2] Hulme A. The heave added-mass and damping coefficients of a submerged torus. J. Fluid Mechanics, $155: 511-530,1985$.

[3] Jennings A. Added mass for fluid-structure vibration problems. Int. J. for Num. Meth. in Engng., $5: 817-830,1985$.

[4] Papanikolau A. On the integral-equation-methods for the evaluation of motions and loads of arbitrary bodies in waves. Ingenieur-Archiv, 55:17-29, 1985.

[5] Davis A.M. Short surface waves due to an oscillating half-inmersed sphere. Mathematika, 18:20-39, 1971.

[6] Lloyd A.R.J.M. Seakeeping. Ship Behavior in Rough Weather. Ellis Horwood Limited (Chichester), 1989.

[7] Tasai F. On the damping force and added mass of ships heaving and pitching. Journal of Zosen Kiokai, 105:47-56, 1959.

[8] Ursell F. On the heave motion of a circular cylinder on the surface of a fluid. Quarterly Journal of Mechanics and Applied Mathematics, 2:218-231, 1949.

[9] Ursell F. Short surface waves due to an oscillatin inmersed body. Proc. R. Soc. Lond. A, 220:90-103, 1953.

[10] Lewis F.M. The inertia of the water surrounding a vibrating ship. In Trans. Soc. Nav. Arch., 37, pages $1-20,1929$

[11] Korsmeyer F.T. and Sclavounos P.D. The large-time asymptotic expansion of the impulse response function for a floating body. Applied Ocean Research, 11(2):75-88, 1989.

[12] Nicholls F.W. Vibration of ships. In Trans RINA, 66, pages 141-163, 1924.

[13] Lamb H. Hydrodynamics. Dover Pubns, 6th edition, 1993.

[14] Prins H.J. Time-domain Calculations of Drift Forces and Moments. PhD thesis, Technische Universiteit Delft, 1995.

[15] D'Elía J. and Storti M. A kelvin-source mixed computation in ship hydrodynamics. Technical report, CIMEC, 2000.

[16] D'Elía J., Storti M., Oñate E., and Idelsohn S. A nonlinear panel method in the time domain for seakeeping flow problems. International Journal of Computational Fluid Dynamics, 16(4):263-275, 2002. 
[17] D'Elía J., Storti M., and Idelsohn S. A panel-Fourier method for free surface methods. Journal of Fluids Engineering, 122(2):309-317, June 2000.

[18] D'Elía J., Storti M., and Idelsohn S. Iterative solution of panel discretizations for potential flows. the modal/multipolar preconditioning. Int. J. Num. Meth. Fluids, 32(1):1-27, 2000.

[19] Falnes J. and McIver P. Surface wave interactions with system of oscillating bodies and pressure distributions. Applied Ocean Research, 7(4):225-234, 1985.

[20] Stoker J.J. Water Waves. Interscience Publishers, 1957.

[21] Newman J.N. Marine Hydrodynamics. The MIT Press, Cambridge, 1977.

[22] Landweber L. Handbook of Fluid Dynamics, chapter Motion of Immersed and Floating Bodies. McGraw-Hill, 1961.

[23] Sierevogel L. Time-domain Calculations of Ship Motions. PhD thesis, Technische Universiteit Delft, 1998.

[24] Ohkusu M. Advances in Marine Hydrodynamics. Computational Mechanics Publications, 1996.

[25] Storti M., D’Elía J., Bonet R., Nigro N., and Idelsohn S. The DNL absorbing boundary condition. applications to wave problems. Comp. Meth. in App. Mech. and Engng., 182(3-4):483-498, 2000.

[26] Storti M., D'Elía J., and Idelsohn S. Algebraic Discrete Non-Local (DNL) absorbing boundary condition for the ship wave resistance problem. J. Comp. Physics, 146(2):570-602, 1998.

[27] Storti M., D’Elía J., and Idelsohn S. Computing ship wave resistance from wave amplitude with the dnl absorbing boundary condition. Comm. Numer. Meth. Engng., 14:997-1012, 1998.

[28] Grim O. Berechnung der durch Schwingungen eines Schiffskoerpers Erzeugten Hydrodynamischen Kraefte. Jahbuch der Schiffsbautechnischen Gesellshaft, 47:277-299, 1953.

[29] Octave 2.1.33 General Public License, GNU project, i386-redhat-linux-gnu, 2001.

[30] Liapis S.J. Time-domain Analysis of Ship Motions. PhD thesis, University of Michigan, 1986.

[31] Ogilvie T.F. First and second order forces on a cylinder submerged under a free surface. J. of Fluid Mechanics, 16:451-472, 1963.

[32] Ogilvie T.F. Singular-perturbation problems in ship hydrodynamics. Advances in Applied Mechanics, $17: 91-188,1977$. 
[33] Havelock T.H. Waves due to a floating hemi-sphere making periodic heaving oscillations. Proc. R. Soc. Lond. A, 231:1-7, 1955.

[34] Frank W. Oscillation of cylinders in or below the free surface of deep fluids. Technical report, Naval Ship Research and Development Center, 1967.

[35] Porter W.R. Pressure distributions, added mass and damping coefficients for cylinders oscillating in a free surface. Technical Report Report 82-16, University of California, Institute of Engineering Research, Berkeley, 1960.

[36] Huang Y. and Sclavounos P.D. Nonlinear ship motions. J. of Ship Research, 42(2):120-130, 1998. 


\section{List of Figures}

$1 \quad$ Sketch of the movement of an incompressible fluid in ship-like vibration due to a

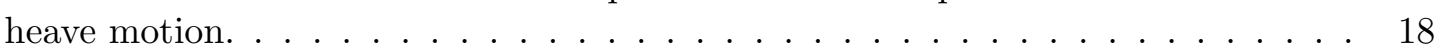

$2 \quad$ Geometrical description of a seakeeping-like flow: original problem (left) and extended to the upper plane (right). $\ldots \ldots \ldots \ldots \ldots$. . . . . . . . . . . . . .

$3 \quad$ Cartesian coordinates $(x, y, z)$ and spherical ones $(r, \theta, \varphi) . \ldots \ldots \ldots$

$4 \quad$ The heave load $h(\theta, \varphi)=\cos \theta$, with $0 \leq \theta \leq \pi / 2 . \ldots \ldots \ldots \ldots \ldots$. . . . . . 21

$5 \quad$ The surge load $h(\theta, \varphi)=\sin \varphi \sin \theta$, with $0 \leq \varphi<2 \pi$ and $0 \leq \theta<\pi / 2$. . . . . . 22

$6 \quad$ Symmetrical load extension $h=|\cos \theta|$ for the heave-mode at very-low frequencies

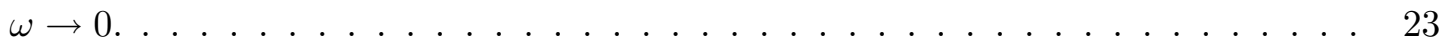

7 Antisymmetrical load extension $h=\cos \theta$ for the heave-mode at very-high fre-

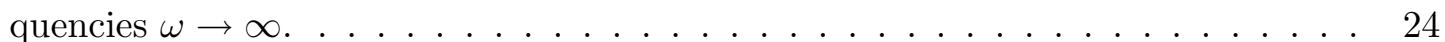

8 The load extension $h=\sin \varphi \sin \theta$ for the surge-mode at Very Low Frequencies

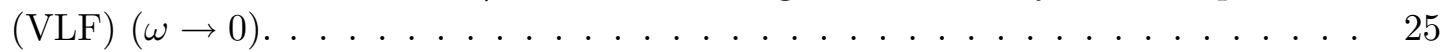

$9 \quad$ The load extension $h=\sin \varphi \sin \theta \operatorname{sign}\{\cos \theta\}$ for the surge-mode at Very High Frequencies $(\mathrm{VHF})(\omega \rightarrow \infty) . \ldots \ldots \ldots \ldots \ldots$ 


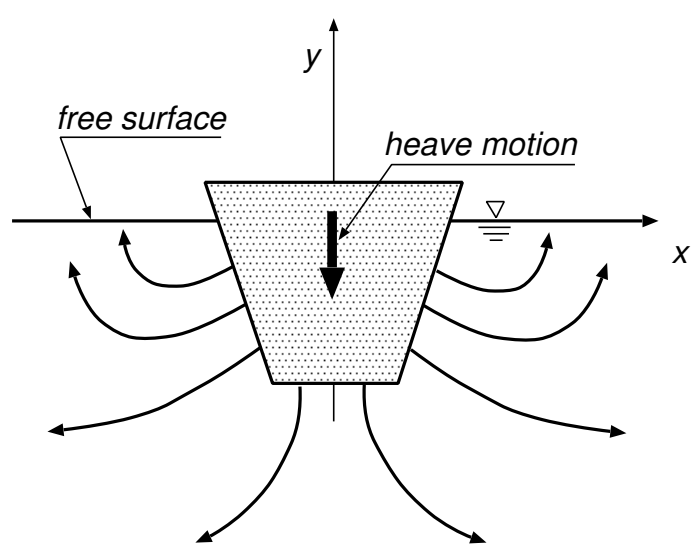

Figure 1: Sketch of the movement of an incompressible fluid in ship-like vibration due to a heave motion. 

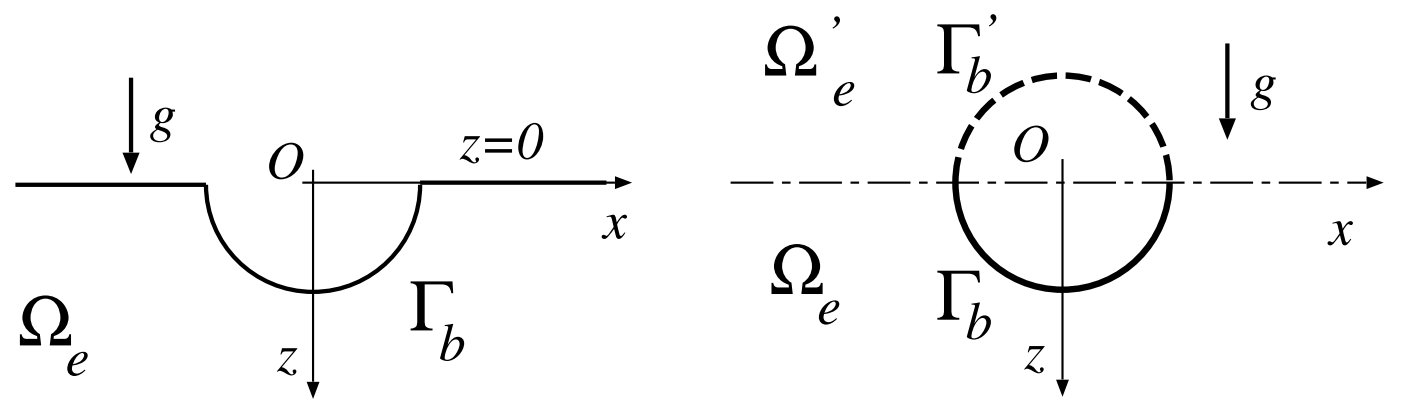

Figure 2: Geometrical description of a seakeeping-like flow: original problem (left) and extended to the upper plane (right). 


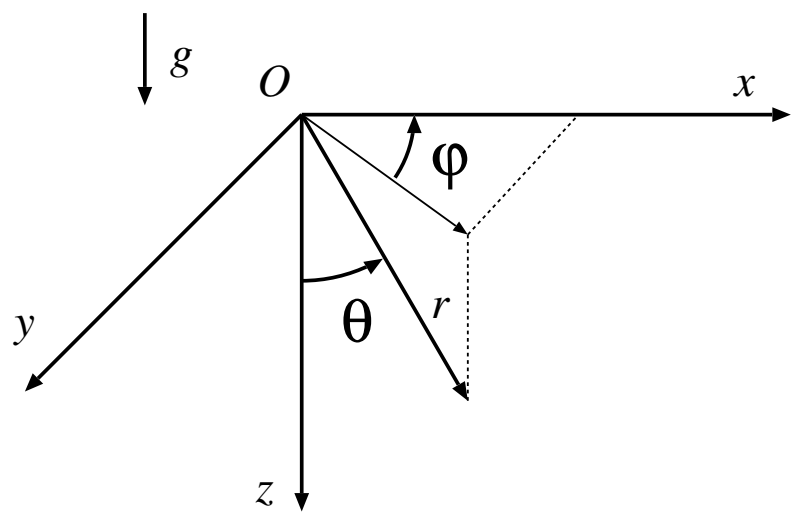

Figure 3: Cartesian coordinates $(x, y, z)$ and spherical ones $(r, \theta, \varphi)$. 

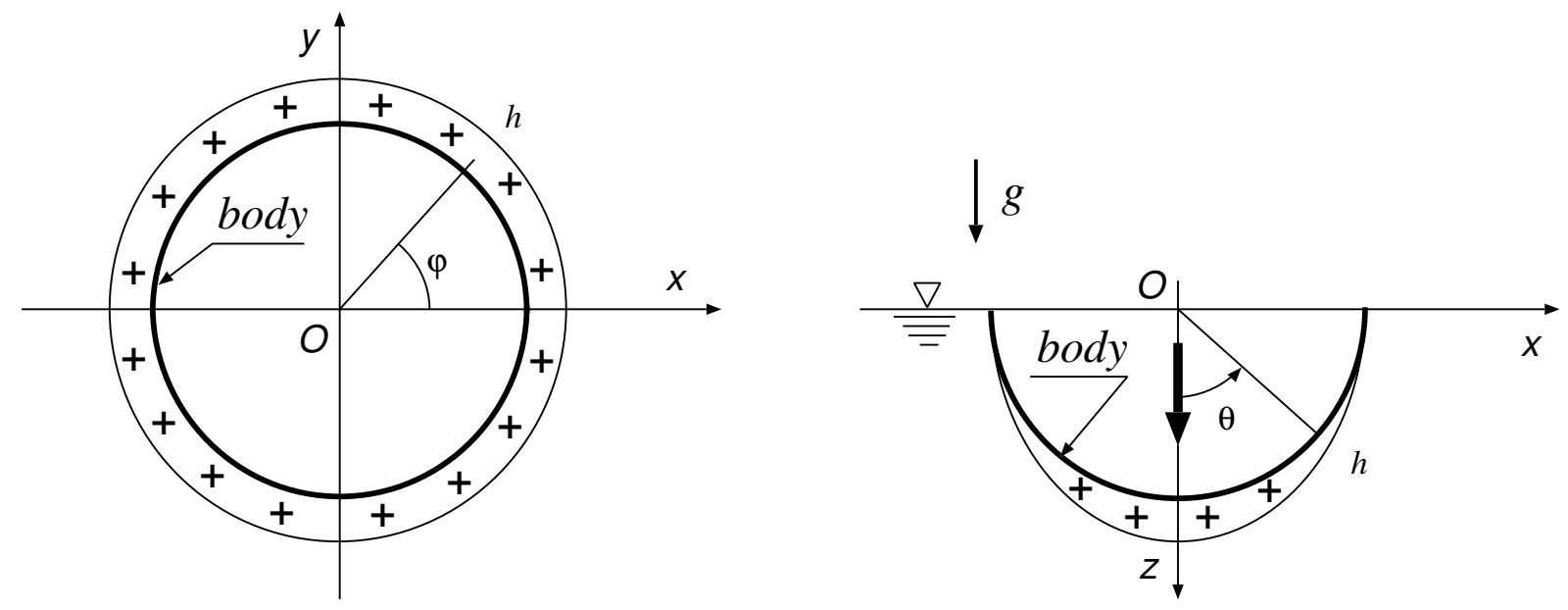

Figure 4: The heave load $h(\theta, \varphi)=\cos \theta$, with $0 \leq \theta \leq \pi / 2$. 

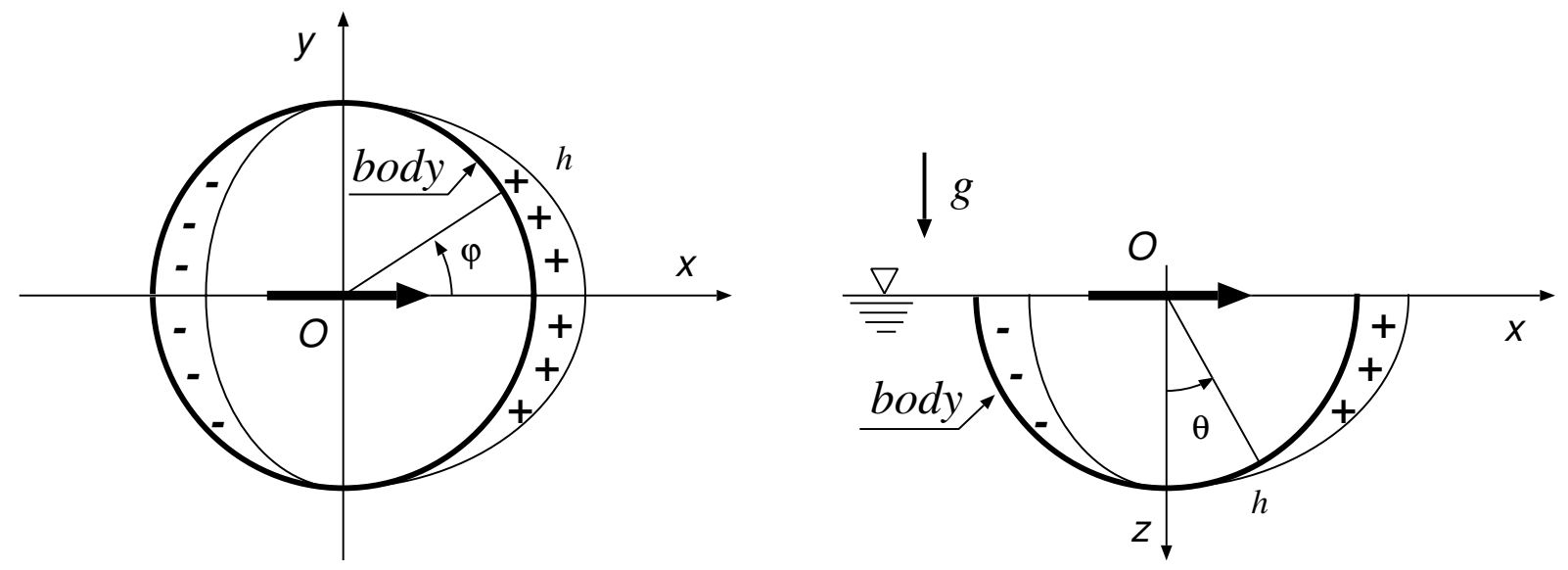

Figure 5: The surge load $h(\theta, \varphi)=\sin \varphi \sin \theta$, with $0 \leq \varphi<2 \pi$ and $0 \leq \theta<\pi / 2$. 


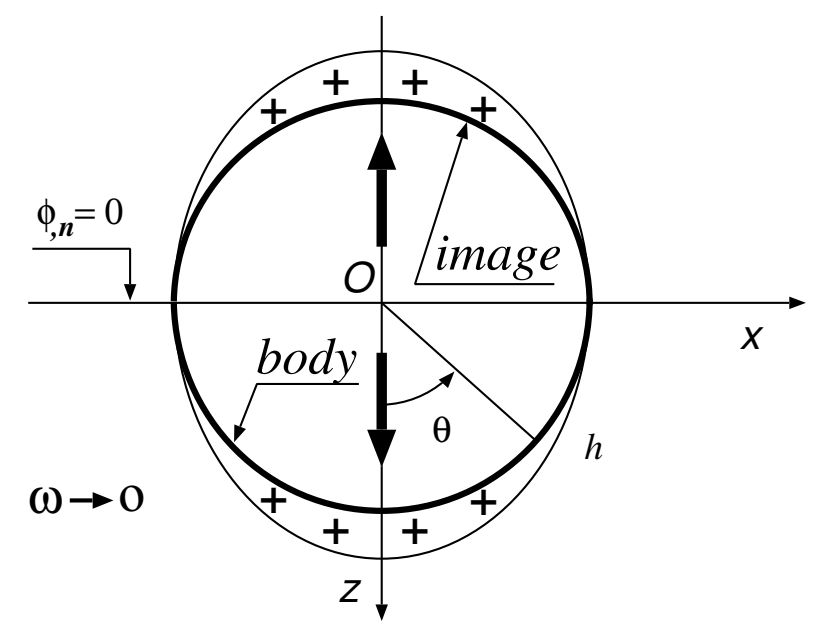

Figure 6: Symmetrical load extension $h=|\cos \theta|$ for the heave-mode at very-low frequencies $\omega \rightarrow 0$. 


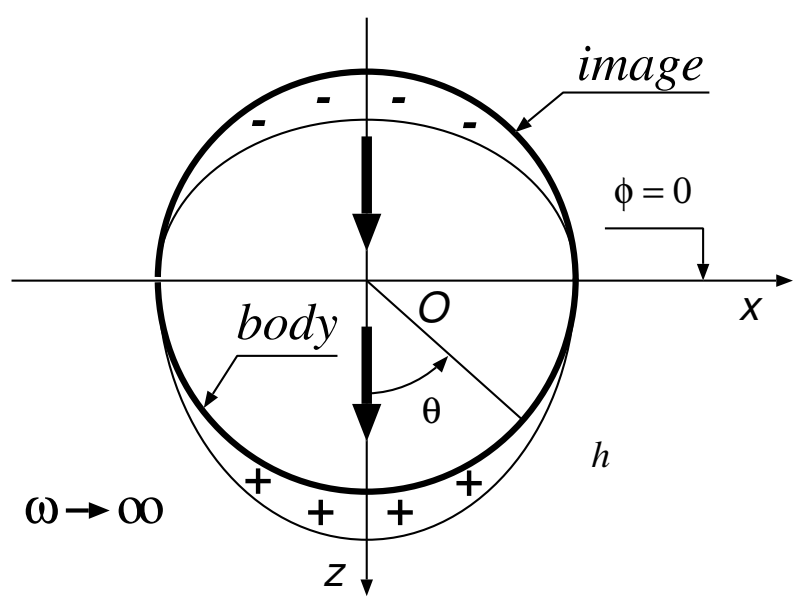

Figure 7: Antisymmetrical load extension $h=\cos \theta$ for the heave-mode at very-high frequencies $\omega \rightarrow \infty$. 


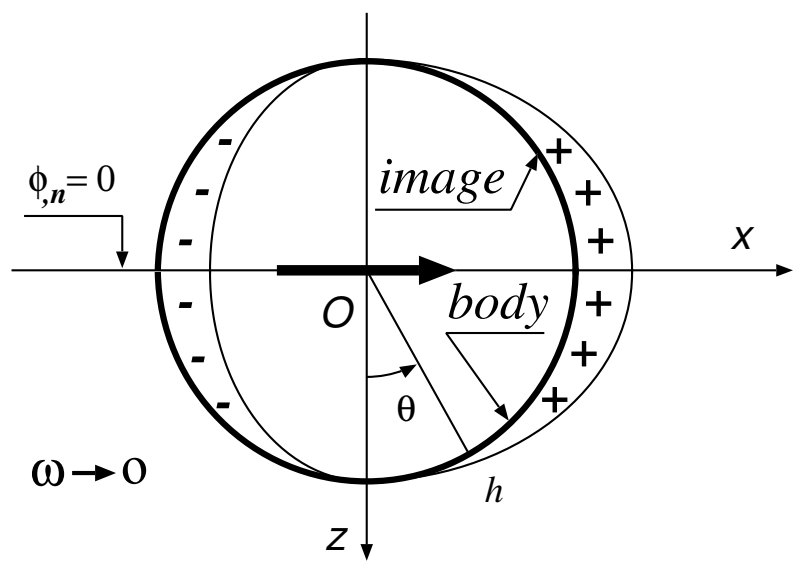

Figure 8: The load extension $h=\sin \varphi \sin \theta$ for the surge-mode at Very Low Frequencies (VLF) $(\omega \rightarrow 0)$. 


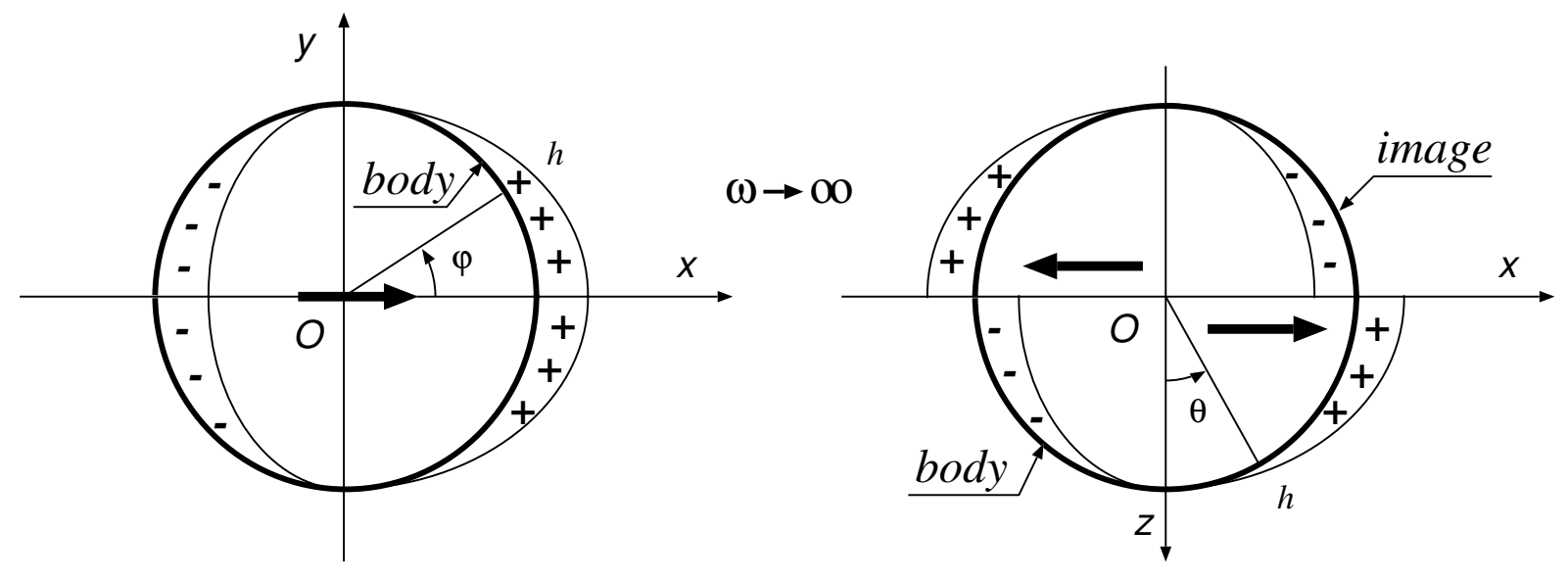

Figure 9: The load extension $h=\sin \varphi \sin \theta \operatorname{sign}\{\cos \theta\}$ for the surge-mode at Very High Frequencies (VHF) $(\omega \rightarrow \infty)$. 


\section{List of Tables}

$1 \quad$ Added mass at Very-Low Frequencies (VLF) $(\omega \rightarrow 0)$ and Very-High Frequencies (VHF) $(\omega \rightarrow \infty)$ for the surge mode $i=1$ (longitudinal oscillation) and the heave one $i=3$ (vertical oscillation) on the unit hemisphere. . . . . . . . . . . . 28 


\begin{tabular}{|l|l|l|l|l|}
\hline \multicolumn{2}{|c|}{ VLF $(\omega \rightarrow 0)$} & \multicolumn{2}{c|}{ VHF $(\omega \rightarrow \infty)$} & \\
\hline surge & heave & surge & heave & \\
$A_{11}^{\prime}$ & $A_{33}^{\prime}$ & $A_{11}^{\prime}$ & $A_{33}^{\prime}$ & reference \\
\hline 0.5 & 0.8 & 0.20 & 0.40 & Sierevoge $[$ 23, Prins 1 14 \\
0.5 & 0.8 & 0.25 & 0.45 & Korsmeyer \\
0.5 & 0.830951 & 0.273239 & 0.50 & Hulme Liapis \\
0.5 & 0.830930 & 0.272220 & 0.50 & present work \\
\hline
\end{tabular}

Table 1: Added mass at Very-Low Frequencies (VLF) $(\omega \rightarrow 0)$ and Very-High Frequencies (VHF) $(\omega \rightarrow \infty)$ for the surge mode $i=1$ (longitudinal oscillation) and the heave one $i=3$ (vertical oscillation) on the unit hemisphere. 